\title{
Green Growth: A Theoretical Model Based on Schumpeterian Product Vertical Innovation Framework
}

\author{
Chaofan Chen ${ }^{1,2}$ \\ ${ }^{1}$ School of Economics and Resource Management \\ Beijing Normal University \\ ${ }^{2}$ School of Statistics \\ Beijing Normal University \\ Beijing, China \\ chaofanc@bnu.edu.cn
}

\author{
Yawen Sun* \\ School of Economics and Resource Management \\ Beijing Normal University \\ Beijing, China \\ yawens1995@163.com
}

\begin{abstract}
The paper constructs an endogenous growth model based on Schumpeterian approach to demonstrate the possibility and conditions of green growth. It is finding that despite the constraints of natural resources and environment, there exis ts an optimal growth path and along this path sustained economic growth with improvement of environmental quality and resources utilization can be achieved. Schumpeterian approach distinguishes intellectual capital and tangible capital, which recognizes the technology for producing knowledge is cleaner than the technology for producing material goods, highlighting the role of innovation in green growth.
\end{abstract}

Keywords-Green growth; Endogenous growth model; Product vertical innovation; Non-renewable resources; Environmental quality; Optimal path

\section{INTRODUCTION}

The "green growth", a term rarely heard before 2008 has burst onto the international policy scene over recent years [1]. Including the organizations of World Bank, OECD, UNEP and some major countries have adopted green growth as an explicit policy objective. In fact, the concept of green growth comes from sustainable development which can be treated as a short to medium term strategy or tool to achieve the Sustainable Development Goals (SDGs). Although there is no consistent definition of green growth in the world [2-4], the core meaning of green growth can be simply stated. It is a new economic growth pattern which also achieves significant environmental protection and improves social well-being.

Until now, green growth is more used as a concept or a strategy appears in the policy discourse. In academic fields, although some quantitative studies have been carried out around the green growth [5-7], there is still lack of a proper theoretical model to explain green growth. In fact, adding the role of depleted nature resources and the cost of environmental pollution into the social welfare function would be a good framework to explain sustainability issues [8]. Compared with neoclassical growth theory, the problems of sustainable development can be better interpreted by endogenous growth theory [9-11]. As a part of sustainable development, green growth must catalyze investment and innovation which will underpin sustained growth and give rise to new economic opportunities [3]. Based on this, we construct an endogenous growth model under the constraints of non-renewable resources and environmental pollution based on the Schumpeterian product vertical innovation framework to demonstrate the possibility and conditions of green growth.

\section{THE MODEL}

According to the simplified model developed by Aghion and Howitt [9] under the Schumpeterian product vertical innovation framework, the production function is obtained:

$$
Y=L^{1-\alpha} \int_{0}^{1} B(i) x(i)^{\alpha} d i
$$

Assume that the final output is produced using labor and a continuum of different intermediate goods. The $L$ is the amount of labor and $x(i)$ is the amount of intermediate good $i$. Each intermediate good is produced according to $x(i)=K(i) / B(i)$, where $K(i)$ is the amount of capital and $B(i)$ is the productivity of intermediate good measured by quality parameter. The $x(i)=x=K / B$ indicates that it is optimal to produce the same quantity of each intermediate good, and $B$ is the average quality.

Since $\int_{0}^{1} B(i) x(i)^{\alpha} d i=x^{\alpha} \int_{0}^{1} B(i) d i=(K / B)^{\alpha} B$, production function can be written as:

$$
Y=F(K, B L)=K^{\alpha}(B L)^{1-\alpha}=K^{\alpha} B^{1-\alpha}(1-n)^{1-\alpha}
$$

Where $n$ represents the amount of labor used in $R \& D$ department, and suppose that the economy-wide frequency of innovations is proportional to the amount of R\&D: $\eta n$, where $\eta$ is a positive parameter of the technology. $B^{\max }$ denotes the maximum of all existing $B(i)$ which is also known as the leading-edge technology parameter. Assume that each innovation has the same probability occurring in any sector, so in the long run, there is a strict proportional relationship 
between the leading-edge parameter and the average parameter: $B^{\max }=(1+v) B$, where $v$ is the technological frontier rate, and it follows that: $\dot{B}=v \eta n B$.

Environmental quality and non-renewable natural resources can be added into the above framework. Environmental quality $E$ should be regarded as a kind of capital good. $E$ is depleted over time by pollution, and the pollution flow $P$ is a function of output and pollution intensity $z: P(Y, z)=Y z^{\gamma}$, where $\gamma>0$, which can be interpreted as the stringency of environmental standards. Meanwhile, environment can self-repair within the acceptable range. $\theta$ represents the self-purification capacity, $\theta>0$. We set an upper limit of environmental quality, which is the highest environmental quality when all economic activities are stopped indefinitely. Since the actual economic activities cannot stop indefinitely, the difference between the actual environmental quality and the upper limit of environmental quality limit is always negative:

$$
\dot{E}=-P(Y, z)-\theta E=-Y z^{\gamma}-\theta E
$$

Meanwhile, there is a lower limit of environmental quality $E^{\mathrm{min}}$, it can be considered as the ecological threshold. According to the nonpositivity of $E$, the optimal growth path must obey a constraint: $E^{\min } \leq E(t) \leq 0$, for all $t$. For nonrenewable natural resources, assume that the stock of resources is $S, S(0)>0$, and its rate of change is negatively correlated with the flow $R$ of resources extraction, hence $\dot{S}=-R$.

Based on this, we incorporate $z$ and $R$ into the production function: $Y=K^{\alpha} B^{1-\alpha}(1-n)^{\beta} R^{\delta} z$, where $\alpha, \beta$ and $\delta$ are all positive, and $\alpha+\beta+\delta=1$.

Assume that the difference between output $Y$ and consumption $C$ is equal to the change of tangible capital: $\dot{K}=Y-C$. Assume that under infinite-horizon the utility of a representative consumer depends on $C$ and $E$. A utility function which has an additive isoelastic form can be constructed:

$$
U(C, E)=\frac{C^{1-\sigma}-1}{1-\sigma}+\frac{-\left[(-E)^{1+\omega}-1\right]}{1+\omega} \quad \sigma>1, \quad \omega>0
$$

The $U(C, E)$ denotes the instantaneous utility function. $\sigma$ is the elasticity of marginal utility, and $\omega$ is the degree of social preference for environmental quality, i.e. the parameter of environmental awareness.

\section{Optimal GROWTH PATH AND CONDITIONS}

Based on the above green growth model, we can find the optimal growth path by maximizing the utility function:

$$
\left\{\begin{array}{l}
\max \int_{0}^{\infty} e^{-\rho t} U(C, E) d t \quad U(C, E)=\frac{C^{1-\sigma}-1}{1-\sigma}+\frac{-\left[(-E)^{1+\omega}-1\right]}{1+\omega} \\
\text { s.t. } \quad \dot{K}=Y-C \quad Y=K^{\alpha} B^{1-\alpha}(1-n)^{\beta} R^{\delta} z \\
\dot{B}=v \eta n B \\
\dot{E}=-Y z^{\gamma}-\theta E \\
\dot{S}=-R \\
\alpha+\beta+\delta=1
\end{array}\right.
$$

Where, $e^{-\rho t}$ is a discount factor and $\rho$ is a positive time preference rate. The $C, n, z$ and $R$ are control variables, and $K$, $B, E$ and $S$ are state variables.

Using Pontryagin maximum principle to construct the current Hamilton function of the optimal growth path:

$$
H=U(C, E)+\lambda_{1} \dot{K}+\lambda_{2} \dot{B}+\lambda_{3} \dot{E}+\lambda_{4} \dot{S}
$$

$$
H=U(C, E)+\lambda_{1}(Y-C)+\lambda_{2}(v \eta n B)+\lambda_{3}\left(-Y z^{\gamma}-\theta E\right)+\lambda_{4}(-R)
$$

Where, $\lambda$ denotes the shadow price. Make the first-order derivative of the control variables equal to zero:

$$
\begin{aligned}
& \left\{\begin{array}{l}
\frac{\partial H}{\partial C}=0 \Rightarrow C^{-\sigma}-\lambda_{1}=0 \\
\frac{\partial H}{\partial n}=0 \Rightarrow \frac{Y \beta}{1-n} \lambda_{1}-v \eta B \lambda_{2}-\frac{z^{\gamma} Y \beta}{1-n} \lambda_{3}=0 \\
\frac{\partial H}{\partial z}=0 \Rightarrow \frac{Y}{z} \lambda_{1}-\frac{(1+\gamma) z^{\gamma} Y}{z} \lambda_{3}=0 \\
\frac{\partial H}{\partial R}=0 \Rightarrow \frac{\delta Y}{R} \lambda_{1}-\frac{z^{\gamma} \delta Y}{R} \lambda_{3}-\lambda_{4}=0
\end{array}\right. \\
& \left\{\begin{array}{l}
\lambda_{1}=C^{-\sigma} \\
\lambda_{2}=\frac{Y \beta \gamma}{(1-n)(1+\gamma) v \eta B} \lambda_{1} \\
\lambda_{3}=\frac{1}{(1+\gamma) z^{\gamma}} \lambda_{1} \\
\lambda_{4}=\frac{\delta \gamma Y}{R(1+\gamma)} \lambda_{1}
\end{array}\right.
\end{aligned}
$$

Set the growth rate of each variable is: $g_{X}=\dot{X} / X$, and take the logarithm of the two sides of the above equations and deal with the derivative to get:

$$
\left\{\begin{array}{l}
g_{\lambda_{1}}=-\sigma g_{C} \\
g_{\lambda_{2}}=g_{\lambda_{1}}+g_{Y}-g_{B}-g_{n} \\
g_{\lambda_{3}}=g_{\lambda_{1}}-\gamma g_{z} \\
g_{\lambda_{4}}=g_{Y}+g_{\lambda_{1}}-g_{R}
\end{array}\right.
$$

Moreover, the Euler equations of the state variables are as follows: 


$$
\begin{aligned}
& \left\{\begin{array}{l}
\dot{\lambda}_{1}=\rho \lambda_{1}-\frac{\partial H}{\partial K}=\rho \lambda_{1}-\frac{\alpha Y}{K} \lambda_{1}+\frac{z^{\gamma} \alpha Y}{K} \lambda_{3} \\
\dot{\lambda}_{2}=\rho \lambda_{2}-\frac{\partial H}{\partial B}=\rho \lambda_{2}-\frac{(1-\alpha) Y}{B} \lambda_{1}-v \eta n \lambda_{2}+\frac{z^{\gamma}(1-\alpha) Y}{B} \lambda_{3} \\
\dot{\lambda}_{3}=\rho \lambda_{3}-\frac{\partial H}{\partial E}=\rho \lambda_{3}+(-E)^{\omega}+\theta \lambda_{3} \\
\dot{\lambda}_{4}=\rho \lambda_{4}-\frac{\partial H}{\partial S}=\rho \lambda_{4}
\end{array}\right. \\
& \left\{\begin{array}{l}
\dot{\lambda}_{1}=\left[\rho-\frac{\alpha Y \gamma}{K(1+\gamma)}\right] \lambda_{1} \\
\dot{\lambda}_{2}=\left[\rho-v \eta n-\frac{v \eta(1-\alpha)(1-n)}{\beta}\right] \lambda_{2} \\
\dot{\lambda}_{3}=\left[\rho+\theta-\frac{(-E)^{\omega}}{\lambda_{3}}\right] \lambda_{3} \\
\dot{\lambda}_{4}=\rho \lambda_{4}
\end{array}\right.
\end{aligned}
$$

The transversality conditions are:

$$
\begin{cases}\lim _{t \rightarrow \infty} \lambda_{1} K e^{-\rho t}=0 & \lim _{t \rightarrow \infty} \lambda_{2} B e^{-\rho t}=0 \\ \lim _{t \rightarrow \infty} \lambda_{3} E e^{-\rho t}=0 & \lim _{t \rightarrow \infty} \lambda_{4} S e^{-\rho t}=0\end{cases}
$$

Meanwhile, according to the Euler equations, we can get:

$$
\left\{\begin{array}{l}
g_{\lambda_{1}}=\rho-\frac{\alpha Y \gamma}{K(1+\gamma)} \\
g_{\lambda_{2}}=\rho-v \eta n-\frac{v \eta(1-\alpha)(1-n)}{\beta} \\
g_{\lambda_{3}}=\omega g_{E} \\
g_{\lambda_{4}}=\rho
\end{array}\right.
$$

According to the production function, the $g_{C}=\alpha g_{C}+(1-\alpha) g_{B}+\beta g_{n}+\delta g_{R}+g_{z}$ is obtained, and according to $\dot{B}=v \eta n B, g_{B}=v \eta n$ is obtained. Since $g_{B}$ is constant, $g_{n}=0$. According to $\dot{E}=-Y Z^{\gamma}-\theta E, g_{E}=g_{C}+\gamma g_{Z}$ is obtained, and according to $\dot{S}=-R, g_{S}=g_{R}$ is obtained.

Combined with the above mathematical induction and analysis, we can get:

$$
\begin{aligned}
& \left\{\begin{array}{l}
g_{\lambda_{1}}=-\sigma g_{C} \\
g_{\lambda_{3}}=g_{\lambda_{1}}-\gamma g_{z} \quad \Rightarrow \omega g_{E}=-\sigma g_{C}-\gamma g_{z} \\
g_{\lambda_{3}}=\omega g_{E}
\end{array}\right. \\
& \left\{\begin{array}{l}
g_{\lambda_{1}}=-\sigma g_{C} \\
g_{\lambda_{4}}=g_{Y}+g_{\lambda_{1}}-g_{R} \quad \Rightarrow g_{R}=(1-\sigma) g_{C}-\rho \\
g_{\lambda_{4}}=\rho
\end{array}\right.
\end{aligned}
$$

$$
\begin{aligned}
& \left\{\begin{array}{l}
g_{C}=\alpha g_{C}+(1-\alpha) g_{B}+\beta g_{n}+\delta g_{R}+g_{z} \\
g_{n}=0 \\
g_{B}=v \eta n \\
g_{R}=(1-\sigma) g_{C}-\rho \\
g_{z}=-\frac{(\omega+\sigma)}{\gamma(1+\omega)} g_{C}
\end{array}\right. \\
& \Rightarrow(1-\alpha) g_{C}=(1-\alpha) v \eta n+\delta\left[(1-\sigma) g_{C}-\rho\right]-\frac{(\omega+\sigma)}{\gamma(1+\omega)} g_{C} \\
& \qquad \begin{array}{l}
g_{\lambda_{1}}=-\sigma g_{C} \\
g_{\lambda_{2}}=g_{\lambda_{1}}+g_{Y}-g_{B}-g_{n} \\
g_{\lambda_{2}}=\rho-v \eta n-\frac{v \eta(1-\alpha)(1-n)}{\beta}
\end{array} \\
& \Rightarrow g_{C}=\frac{1}{1-\sigma}\left[\rho-\frac{v \eta(1-\alpha)(1-n)}{\beta}\right]
\end{aligned}
$$

The growth path can be defined recursively, and then we get the equilibrium growth rate of each variable:

$$
\left\{\begin{array}{l}
g_{n}=0 \\
g_{C}=g_{Y}=g_{K}=(v \eta-\rho)\left[\sigma+\frac{(\sigma+\omega) /(1+\omega)}{\gamma(1-\alpha)}\right]^{-1} \\
g_{E}=g_{C}+\gamma g_{Z}=\frac{1-\sigma}{1+\omega} g_{C} \\
g_{z}=-\frac{(\omega+\sigma)}{\gamma(1+\omega)} g_{C} \\
g_{B}=\left[1+\frac{(\sigma+\omega) /(1+\omega)}{\gamma(1-\alpha)}\right] g_{C} \\
g_{S}=g_{R}=(1-\sigma) g_{C}-\rho \\
g_{\lambda_{1}}=-\sigma g_{C} \\
g_{\lambda_{2}}=(1-\sigma) g_{C}-g_{B} \\
g_{\lambda_{3}}=\omega\left(\frac{(1-\sigma)}{1+\omega}\right) g_{C} \\
g_{\lambda_{4}}=\rho
\end{array}\right.
$$

More specifically, we find that if the critical conditions are satisfied:

$$
\left\{\begin{array}{l}
(v \eta-\rho)>0 \\
(\sigma-1)>0 \\
(1-\sigma)(v \eta-\rho)\left[\sigma+\frac{(\sigma+\omega) /(1+\omega)}{\gamma(1-\alpha)}\right]^{-1}-\rho<0 \\
(\sigma-1)(v \eta-\rho)<\theta\left[\sigma(1+\omega)+\frac{\sigma+\omega}{(1-\alpha) \gamma}\right]
\end{array}\right.
$$

Then for any initial level of environmental quality $E_{0}$ and resources $S_{0}$, there exists a pair of hypothetical initial values of $K_{0}$ and $B_{0}$ so that we can get the optimal growth path from 
the initial state to the equilibrium state, and under this path the growth will be unrestricted.

Through the above analysis, we can define the optimal path: $g_{Y}=g_{K}=g_{C}>0, g_{E}<0, g_{S}<0$. Under this path, the economic growth rate is positive and the growth rate of environmental pollution and resource consumption is negative. In order to achieve this optimal equilibrium state, the following four conditions should be satisfied. First, $(v \eta-\rho)>0$. This condition indicates that as long as the productivity of intellectual capital is larger than the time discount rate, i.e. giving full play to the promotion effect of innovation on green growth, then the long-term growth can be achieved. Second, $(\sigma-1)>0$, which is a necessary condition for the improvement of environmental quality. It ensures that $g_{E}<0$, i.e. rational consumers will not destroy the environmental quality under the threshold. Third, $(1-\sigma)(v \eta-\rho)<0$, which guarantees $g_{s}<0$. It means that the extraction rate of nonrenewable resources is negative. Fourth, when the selfpurification capacity of the environment $\theta$ is large enough, the excessive growth of pollution relative to environmental regeneration can be avoided.

We can make a comparative static analysis of the key parameters to explore the dynamic mechanism of green growth under the optimal path, see Table 1.

TABLE I. COMPARATIVE ST ATIC ANALYSIS

\begin{tabular}{|c|c|c|c|c|c|c|}
\hline & $\mu=v$ & $\mu=\eta$ & $\mu=\rho$ & $\mu=\sigma$ & $\mu=\omega$ & $\mu=\gamma$ \\
\hline$\partial g_{Y} / \partial \mu$ & $\partial g_{Y} / \partial \mu>0$ & $\partial g_{Y} / \partial \mu>0$ & $\partial g_{Y} / \partial \mu<0$ & $\partial g_{Y} / \partial \mu<0$ & $\partial g_{Y} / \partial \mu>0$ & $\partial g_{Y} / \partial \mu>0$ \\
\hline$\partial g_{E} / \partial \mu$ & $\partial g_{E} / \partial \mu<0$ & $\partial g_{E} / \partial \mu<0$ & $\partial g_{E} / \partial \mu>0$ & $\partial g_{E} / \partial \mu<0$ & $\partial g_{E} / \partial \mu<0$ & $\partial g_{E} / \partial \mu<0$ \\
\hline$\partial g_{S} / \partial \mu$ & $\partial g_{S} / \partial \mu<0$ & $\partial g_{S} / \partial \mu<0$ & $\partial g_{S} / \partial \mu>0$ & $\partial g_{S} / \partial \mu<0$ & $\partial g_{S} / \partial \mu<0$ & $\partial g_{S} / \partial \mu<0$ \\
\hline
\end{tabular}

First, under the condition of other parameters unchanged (COPU), the greater the technological frontier rate $v$ and technical parameter $\eta$, the higher the growth rate. Second, under the COPU, the smaller the time preference rate $\rho$, the larger the elasticity of intertemporal substitution $1 / \sigma$ ( the smaller the $\sigma$ ) and the larger the environmental awareness parameter $\omega$, the higher the growth rate. The smaller $\rho$ indicates that consumers are more aware of the green growth and are more willing to choose future consumption. The larger $\omega$ indicates the greater social preference for environmental quality. The smaller $\sigma$ shows that consumers have a good intergenerational concept, otherwise, it shows that consumers lack patience and the view of equity between generations. Third, under the COPU, the larger the $\gamma$ (which can reflect the stringency of environmental standards), the higher the steady-state growth rate.

\section{CONCLUSION}

The paper constructs an endogenous growth model based on Schumpeterian product vertical innovation framework to demonstrate the possibility and conditions of green growth when the non-renewable resources and environment quality are used as inputs to production. In the model, Schumpeterian approach distinguishes the intellectual capital and tangible capital, and highlights the role of innovation in green growth. We find that if the accumulation speeds of intellectual capital faster than tangible capital and which is sufficient to offset the irreversible decline of environment quality and resources consumption, the growth of output will be sustained. Under the optimal path, the economic growth rate is positive, and the growth rate of environmental pollution and resource consumption are negative. In order to achieve the optimal equilibrium state, it should meet: the productivity of intellectual capital is greater than the time discount rate, the elasticity of intertemporal substitution is less than 1 , the consumption rate of non-renewable resources is negative, and the parameter of environmental self-purification is large enough. Moreover, stimulating knowledge accumulation and technology innovation, improving consumer's environmental awareness and intergenerational concept, and strengthening environmental supervision will be benefit for promoting the green growth.

\section{REFERENCES}

[1] M. Jacobs, "Green growth: Economic theory and political discourse," Centre for climate change economics and policy working paper, 2012, WP 108-2012.

[2] UNEP, "Towards a green economy: Pathways to sustainable development and poverty eradication-A Synthesis for Policy Makers," UNEP Publishing, Nairobi, 2011.

[3] OECD, “Towards green growth,” OECD Publishing, Paris, 2011.

[4] World Bank, "Inclusive green growth: The pathway to sustainable development,” World Bank Publishing, Washington, DC, 2012.

[5] S. Chen, J. Golley, "Green productivity growth in China's industrial economy,” Energ. Econ. vol.44, pp. 89-98, 2014.

[6] H. Dai, X. Xie, Y. Xie, “Green growth: The economic impacts of largescale renewable energy development in China,” Appl. Energ. vol.162, pp. 435-449, 2016.

[7] M. Antal , J. C. J. M. Van Den Bergh, "Green growth and climate change: Conceptual and empirical considerations,” Clim. Policy. vol.16, pp.165-177, 2016.

[8] P. Dasgupta, "Optimal development and the idea of net national product,” In: Goldin IA, Winters LA (eds) The economics of sustainable development. Cambridge University Press, pp.111-142, 1995.

[9] P. Aghion , P. Howitt, M. Brant-Collett, "Endogenous growth theory," MIT press, Boston, pp.151-169, 1998. 
[10] A.Grimaud, L. Rougé, “Non-renewable resources and growth with vertical innovations: optimum, equilibrium and economic policies,” J. Environ. Econ. Manag.vol.45, pp.433-453, 2003.

[11] S.Peng, Q. Bao, "Environmental pollution, endogenous growth and sustainable development,” Quant. Tech. Econ. vol.23, pp.114-126, 2006. (In Chinese) 